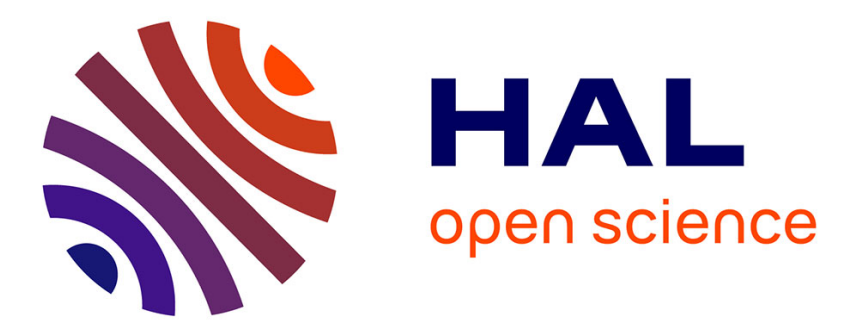

\title{
DTW for Matching Radon Features: A Pattern Recognition and Retrieval Method
}

\author{
Santosh K.C., Bart Lamiroy, Laurent Wendling
}

\section{To cite this version:}

Santosh K.C., Bart Lamiroy, Laurent Wendling. DTW for Matching Radon Features: A Pattern Recognition and Retrieval Method. Advanced Concepts for Intelligent Vision Systems, Aug 2011, Ghent, Belgium. pp.249-260, 10.1007/978-3-642-23687-7_23 . inria-00617287

\section{HAL Id: inria-00617287 \\ https://hal.inria.fr/inria-00617287}

Submitted on 26 Aug 2011

HAL is a multi-disciplinary open access archive for the deposit and dissemination of scientific research documents, whether they are published or not. The documents may come from teaching and research institutions in France or abroad, or from public or private research centers.
L'archive ouverte pluridisciplinaire HAL, est destinée au dépôt et à la diffusion de documents scientifiques de niveau recherche, publiés ou non, émanant des établissements d'enseignement et de recherche français ou étrangers, des laboratoires publics ou privés. 


\title{
DTW for Matching Radon Features: A Pattern Recognition and Retrieval Method
}

\author{
Santosh K.C. ${ }^{1}$, Bart Lamiroy ${ }^{2}$, and Laurent Wendling ${ }^{3}$ \\ 1 INRIA Nancy Grand Est Research Centre, LORIA - Campus Scientifique, \\ BP 239 - 54506 Vandoeuvre-lés-Nancy Cedex, France \\ Santosh.KC@inria.fr \\ 2 Nancy Université INPL, LORIA - Campus Scientifique, \\ BP 239 - 54506 Vandoeuvre-lés-Nancy Cedex, France \\ Bart.Lamiroy@loria.fr \\ 3 LIPADE, Université Paris Descartes \\ 75270 Paris Cedex 06, France \\ Laurent.Wendling@parisdescartes.fr
}

\begin{abstract}
In this paper, we present a method for pattern such as graphical symbol and shape recognition and retrieval. It is basically based on dynamic programming for matching the Radon features. The key characteristic of the method is to use DTW algorithm to match corresponding pairs of histograms at every projecting angle. This allows to exploit the Radon property to include both boundary as internal structure of shapes, while avoiding compressing pattern representation into a single vector and thus miss information, thanks to the DTW. Experimental results show that the method is robust to distortion and degradation including affine transformations.
\end{abstract}

Keywords: Radon Transform, DTW, Pattern Recognition and Retrieval

\section{Introduction}

Given patterns such as symbols of any kind, cursive characters but also forensic elements like human faces, shoe- or finger-prints, recognition or classification can be done using structural [10], statistical [17] as well as hybrid approaches. In structural approaches, graph based methods have been widely used [10]. They provide a powerful relational representation. However, they suffer from intense computational complexity due to the general NP-hard problem of sub-graph matching resulting from the variation of graph structure with the level of noise, occlusion, distortion etc. In some cases, however, matching optimisation can be obtained, but is strongly based on how the image is described [18]. Besides, structural approaches do not have rich set of mathematical tools [7] unlike in statistical approaches [17]. We therefore emphasise in the rest of this paper on statistical pattern analysis representation and recognition. 
Shape representation has been an important issue in pattern analysis and recognition [23,31]. In this context, features are often categorised as regionbased as well as contour-based descriptors. Generally, contour-based descriptors include Fourier Descriptors (FD) [32,12]. Contour information can also come from polygonal primitives [1], or curvature information $[3,5]$. In the case of the latter, the shape is described in the scale space by the maximum number of curvatures. Other methods, like Shape Context (SC) [4] or skeleton approaches [33] are based on contour information. In short, contour-based descriptors are appropriate for silhouette shapes since they cannot capture the interior content as well as disconnected shapes or shapes with holes where boundary information is not available. On the other side, region-based descriptors account all pixels within patterns. Common methods are based on moments [28,2,13] including geometric, Legendre, Zernike, and Pseudo-Zernike moments. Comparative studies [2,28] have demonstrated the interest on improving invariance properties and reducing computational time of the Zernike moments [8]. On the other side, to overcome the drawbacks of contour-based Fourier descriptors, Zhang and Lu [30] have proposed a region-based Generic Fourier Descriptor (GFD). To avoid the problem of rotation in the Fourier spectra, the 2D Fourier Transform (FT) is applied on a polar-raster sampled shape. This approach outperforms common contourbased (classical Fourier and curvature approaches) and region-based (Zernike moments) shape descriptors. Region-based descriptors on the whole, can be generally applied. However, high computational complexity needs to be considered. Besides, use of normalisation in order to satisfy common geometric invariance properties introduces errors as well as they are sensitive to noise, eventually affecting the whole recognition process.

Pattern representation must be sufficiently enriched with important information. Moreover, global pattern representation is the premier choice due to its simplicity that avoids extra pre-processing and segmentation process as in local pattern representation. To accomplish recognition, matching is another concern. In other words, feature selection corresponds to the matching techniques, eventually affects the overall performance of the method. For instance, compressing pattern information into a single vector as in global signal based descriptors provide immediate matching, while not offering complete shape information. In those respects, we take advantage of radon transform [11] to represent pattern and DTW is used to match patterns of any size that avoids compressing pattern representation into a single vector unlike the use of $\mathcal{R}$-transform [27], for instance. The work is inspired from previous works such as $2 D$ shape categorisation [22], gait recognition [6], off-line signature verification [9,24] and orientation estimation as in [16]. We have examined the method over two different datasets: graphical symbol [15] and shape [26].

The remaining of the paper is organised as follows. We start with detailing the proposed method in Section 2, which mainly includes pattern representation and matching. Section 3 provides a series of tests. In Section 4, analysis of the results are observed or examined and discussed thoroughly. The paper is concluded in Section 5 along with future perspectives. 


\section{Method}

In this work, we use the Radon transform to represent patterns [11], Radon based descriptors do not only encode contour information ([14,22], for instance), they also encode internal structure. Radon transforms are essentially a set of parametrized histograms. We apply Dynamic Time Warping to align every histogram for each projecting angle to absorb varying histogram sizes resulting from image signal variations.

\section{$2.1 \quad$ Pattern Representation}

The Radon transform consists of a collection of projections of a pattern at different angles [11]. This is illustrated in 1. In other words, the radon transform of a pattern $f(x, y)$ and for a given set of angles can be thought of as the projection of all non-zero points. This resulting projection is the sum of the non-zero points for the pattern in each direction, thus forming a matrix. The matrix elements are related to the integral of $f$ over a line $L(\rho, \theta)$ defined by $\rho=x \cos \theta+y \sin \theta$ and can formally be expressed as,

$$
R(\rho, \theta)=\int_{-\infty}^{\infty} \int_{-\infty}^{\infty} f(x, y) \delta(x \cos \theta+y \sin \theta-\rho) d x d y
$$

where $\delta($.$) is the Dirac delta function, \delta(x)=1$, if $x=0$ and 0 otherwise. Also, $\theta \in\left[0, \pi[\right.$ and $\rho \in]-\infty, \infty\left[\right.$. For radon transform, $L_{i}$ be in normal form $\left(\rho_{i}, \theta_{i}\right)$. Following Fig. 1 (c), for all $\theta_{i}$, the Radon transform now can be described as the length of intersections of all lines $L_{i}$. Note that the range of $\rho$ i.e., $-\rho_{\min }<\rho \leq$ $\rho_{\max }$ is entirely based on the size of pattern.

Since the Radon transform itself does not satisfy invariance properties, we consider the following affine transformation properties to adapt it for recognition. In case of translation, we use image centroid $\left(x_{c}, y_{c}\right)$ such that translation vector is $\vec{u}=\left(x_{c}, y_{c}\right): R\left(\rho-x_{c} \cos \theta-y_{c} \sin \theta, \theta\right)$. Therefore, translation of $f$ results in the shift of its transform in $\rho$ by a distance equal to the projection of translation vector of the line $L$ (see Fig. 1 (c)). For rotation, we approximate rotation angle orientation as implemented in [16]. Orientation can be estimated as,

$$
\alpha=\arg \left[\min _{\theta} \frac{d^{2} \sigma_{\theta}^{2}}{d \theta^{2}}\right]
$$

where $\sigma_{\theta}^{2}=\frac{1}{P} \sum_{\rho}\left(R(\rho, \theta)-\mu_{\theta}\right)^{2}$ is the variance of projection at $\theta, \mu_{\theta}=$ $\frac{1}{P} \sum_{\rho} R(\rho, \theta)$ and $P$, the number of samples. If angle of rotation is $\alpha$, then $R^{\alpha}(\rho, \theta)=R(\rho, \theta+\alpha)$. This simply implies a circular shift of the histograms such that it does not require histograms duplication from $[0, \pi[$ to $[\pi, 2 \pi[$ as in [9] to make rotation invariant. For scaling, we simply normalise histograms into $[0,1]$ at every projecting angle.

Fig. 2 shows radon features for reference, rotation, scaling, as well as degradation instances from a known class of graphical symbol [15]. In all cases, radon histograms from their corresponding sample images are similar to each other. 


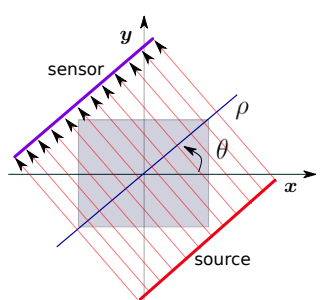

(a) basic projections theory

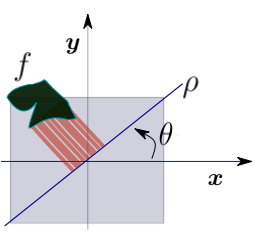

(b) projection at angle $\theta$

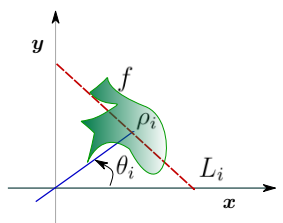

(c) definition

Fig. 1. Radon transform

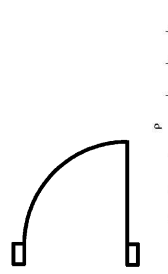

(a) reference

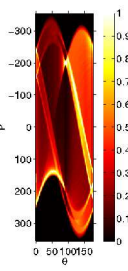

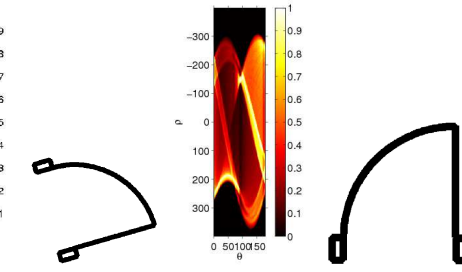

(b) rotation

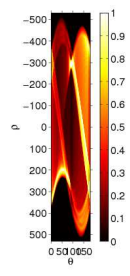

(c) scaling

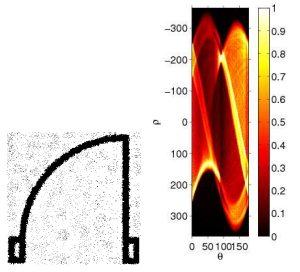

(d) degradation (noise)

Fig. 2. Radon features for ideal (reference), rotation, scaling as well as noisy samples.

\subsection{Pattern Matching}

As explained in Section 2.1, the Radon transform matrix $R(\rho, \theta)$ can represent any pattern $\mathcal{P}$. Given two patterns $\mathcal{P}^{q}$ and $\mathcal{P}^{d}$, matching can be obtained between corresponding histograms $R^{q}(\rho, \theta)$ and $R^{d}(\rho, \theta)$. Radon transforms generate different $\rho$ sizes depending on the image contents' size. In order to be able to adapt to these differences in size, we develop the following approach:

Dynamic Time Warping [20], allows us to find the dissimilarity between two non-linear sequences potentially having different lengths. In radon matrix $R(\rho, \theta)$, column refers to the histogram of radon for each projecting angle. Let us say histograms $\left\{\mathcal{H}_{\theta_{i}}\right\}_{i=0, \ldots, \Theta-1}$ (see Fig. 3). In this illustration, vertical lines represent radon histograms at every projecting angle. Let us consider two column vector sequences from $R(\rho, \theta)$, representing histograms $\mathcal{H}^{q}$ and $\mathcal{H}^{d}$ of length $K$ and $L$, respectively. $\mathcal{H}^{q}=\left\{h_{k}^{q}\right\}_{k=1, \ldots, K}$ and $\mathcal{H}^{d}=\left\{h_{l}^{d}\right\}_{l=1, \ldots, L}$.

At first, a matrix $\mathcal{M}$ of size $K \times L$ is constructed. Then for each element in matrix $\mathcal{M}$, local distance metric $\delta(k, l)$ between the events $e_{k}$ and $e_{l}$ is computed. $\delta(k, l)$ can be expressed as, $\delta(k, l)=\left(e_{k}-e_{l}\right)^{2}$, where $e_{k}=h_{k}^{q}$ and $e_{l}=h_{l}^{d}$. Let $D(k, l)$ be the global distance up to $(k, l)$,

$$
D(k, l)=\min [D(k-1, l-1), D(k-1, l), D(k, l-1)]+\delta(k, l)
$$

with an initial condition $D(1,1)=\delta(1,1)$ such that it allows warping path going diagonally from starting node $(1,1)$ to end $(K, L)$. The main aim is to find the 


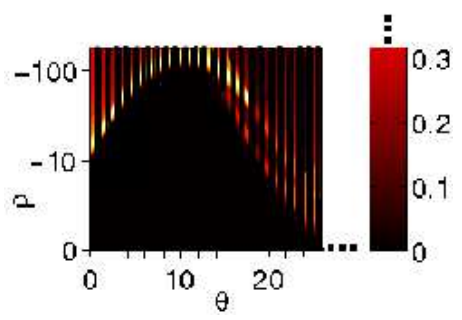

Fig. 3. Radon histogram at every projecting angle $\theta_{i}$.

path for which the least cost is associated. The warping path therefore provides the difference cost between the compared sequences. Formally, the warping path is, $\mathcal{W}=\left\{w_{t}\right\}_{t=1 \ldots T}$ where $\max (k, l) \leq T<k+l-1$ and $t^{t h}$ element of $\mathcal{W}$ is $w(k, l)_{t} \in[1: K] \times[1: L]$ for $t \in[1: T]$. The optimised warping path $\mathcal{W}$ satisfies the following three conditions.

c1. boundary condition: $w_{1}=(1,1)$ and $w_{T}=(K, L)$

c2. monotonicity condition: $k_{1} \leq k_{2} \leq \cdots \leq k_{K}$ and $l_{1} \leq l_{2} \leq \cdots \leq l_{L}$

c3. continuity condition: $w_{t+1}-w_{t} \in\{(1,1)(0,1),(1,0)\}$ for $t \in[1: T-1]$

c1 conveys that the path starts form $(1,1)$ to $(K, L)$, aligning all elements to each other. c2 restricts allowable steps in the warping path to adjacent cells, never be back. And monotonicity condition forces the path advances one step at a time. Note that c3 implies c2.

We then define the global distance between $\mathcal{H}_{q}$ and $\mathcal{H}_{d}$ as,

$$
\Delta\left(\mathcal{H}^{q}, \mathcal{H}^{d}\right)=\frac{D(K, L)}{T} .
$$

The last element of the $K \times L$ matrix, normalised by the $T$ provides the DTWdistance between two sequences where $T$ is the number of discrete warping steps along the diagonal DTW-matrix.

Matching Score Aggregating distances between histograms in all corresponding projecting angles $\theta_{i}$ between $\mathcal{P}^{q}$ and $\mathcal{P}^{d}$ yields a global pattern-matching score,

$$
\operatorname{Dist}\left(\mathcal{P}^{q}, \mathcal{P}^{d}\right)=\sum_{i=0}^{\Theta-1} \Delta\left(\mathcal{H}_{\theta_{i}}^{q}, \mathcal{H}_{\theta_{i}}^{d}\right)
$$

Scores are normalised into $[0,1]$ by, $\overline{\operatorname{Dist}}()=.\frac{\operatorname{Dist}_{(.)}-\operatorname{Dist}^{\min \cdot(.)}}{\operatorname{Dist}^{m a x} \cdot(.)-\operatorname{Dist}^{\min \cdot(.)}}$. As shown in Fig. 2, it is important to notice that there may be significant the amplitude differences between radon histograms, from one sample to another. These amplitude differences are very well handled by the DTW algorithm. In addition, Fig. 4 gives an overview of matching score values of the proposed method to affine transformations, noise addition and stretching. 


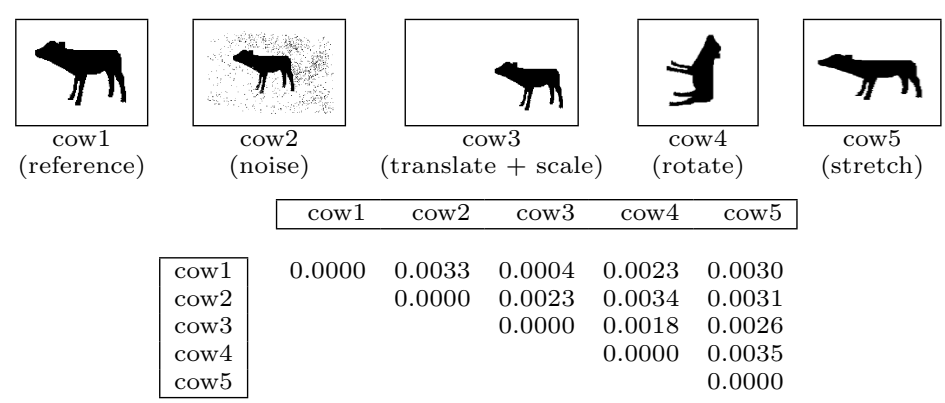

Fig. 4. Matching scores between samples shown above. It provides the differences exist between the samples due to noise, translation, rotation as well as scaling.

\subsection{Pattern Recognition and Retrieval}

We can now use the previously described approach as a global pattern matching score. This score expresses the similarity between database patterns and query. Our problem is: given a set of points $S$ in a metric space $M_{s}$ and a query point $q \in M_{s}$, find the closest point in $S$ to $q$. Now, we express similarity as,

$$
\text { Similarity }\left(\mathcal{P}^{q}, \mathcal{P}^{d}\right)=1-\overline{\operatorname{Dist}}\left(\mathcal{P}^{q}, \mathcal{P}^{d}\right)=\left\{\begin{array}{l}
1 \text { for the closest candidate } \\
0 \text { for the farthest candidate }
\end{array}\right.
$$

Ranking can therefore be expressed on the decreasing order of similarity. In our experiments, we will distinguish "recognition" (search for the closest candidate) from "retrieval" (where closest candidates are retrieved for a given short-list).

\section{$3 \quad$ Experiments}

\subsection{Benchmarking Methods}

We confront $\mathcal{D}$-Radon method with well-known descriptors: $\mathcal{R}$-signature [27], GFD [30], SC [4] and Zernike [19]. For those descriptors, it is important to fit the best parameters. For GFD, we have tuned the parameters, radial (4:12) and angular $(6: 20)$ frequencies to get the best combinations. For SC, we follow [4]. In case of Zernike, we have used 36 Zernike functions of order less than or equal to 7 . For radon, projecting range is $[0, \pi[$.

\subsection{Experimental Results}

We work on several different datasets in different contexts. However, we primarily focus on

A. distorted and degraded symbols in document analysis - graphics recognition and retrieval, and then

B. shape retrieval as a CBIR application. 
In order to test the robustness of the methods, we work on raw data, no prefiltering (de-noising, for instance) has been applied.

\section{A. Symbol Recognition and Retrieval}

GREC2003 dataset symbol recognition contest [15] - In this dataset, we have used the following different categories: ideal, scaling, distortion as well as degradation. Altogether, there are 50 different model symbols. Those symbols are grouped into 3 sets, containing 5, 20 and 50 model symbols. Each model symbol has 5 test images in every category except the ideal one. Ideal test images are directly taken from the set of model symbols and therefore the test is to evaluate the ability of simple shape discrimination, as the number of symbols increases. Since vectorial distortion works only with symbols with straight lines, and not arcs, it is applied to a subset of 15 model symbols. Besides, there are 9 models of degradation, aiming to evaluate the robustness to the scalability with degradation. Fig. 5 shows a few samples of GREC2003 dataset.

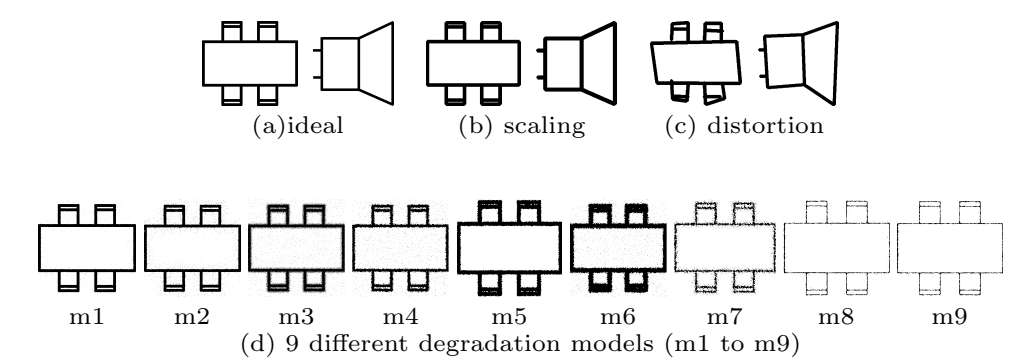

Fig. 5. GREC2003 samples - graphical symbol.

To evaluate the method, each test image is matched with the model symbols to get the closest model. Experimental results for all types of aforementioned categories of datasets are shown in Table 1 . Note that since there are 9 models of degradations ( $\mathrm{m} 1$ to $\mathrm{m} 9$ ), there are nine tests in every sample in each set (see Fig. $5(\mathrm{~d}))$.

Based on the results from ideal test images, one cannot judge the superiority of the methods. Only running time comparison would be an alternative. For scaled images, $\mathcal{R}$-signature lags far behind. Our method achieves $100 \%$ recognition rate while not offering substantial difference with GFD, SC and Zernike. Also, results from test images with vectorial distortions shows identical behaviour as in scaled as well as rotated samples. However, we receive noteworthy differences in case of binary degradations. Overall, $\mathcal{D}$-Radon performs the best of all.

CVC hand-drawn symbol dataset - As in [29], we have used $10 \times 300$ sample images i.e., 10 different known classes of hand-drawn architectural symbols with 300 instances in each. Samples are having distortions, gaps, overlapping as well 
Table 1. Recognition rate in \% for GREC2003 dataset

\begin{tabular}{|c|c|c|c|c|c|c|c|}
\hline & & Test images & $\overline{\mathcal{R} \text {-sign. }}$ & GFD & Zernike & $\mathrm{SC}$ & $\mathcal{D}$-Radon \\
\hline \multirow{3}{*}{ 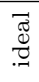 } & set1 & $5 \times 1$ & 100 & 100 & 100 & 100 & 100 \\
\hline & set2 & $20 \times 1$ & 100 & 100 & 100 & 100 & 100 \\
\hline & set3 & $50 \times 1$ & 100 & 100 & 100 & 100 & 100 \\
\hline \multicolumn{3}{|c|}{ Average - ideal } & 100 & 100 & 100 & 100 & 100 \\
\hline \multirow{3}{*}{ 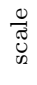 } & set1 & $5 \times 5$ & 45 & 100 & 100 & 100 & 100 \\
\hline & set2 & $20 \times 5$ & 36 & 100 & 98 & 100 & 100 \\
\hline & set3 & $50 \times 5$ & 28 & 98 & 96 & 98 & 100 \\
\hline \multicolumn{3}{|c|}{ Average - scale } & 37 & 99 & 98 & 99 & 100 \\
\hline \multirow{6}{*}{ 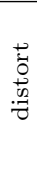 } & distort1-set1 & $5 \times 5$ & 20 & 100 & 100 & 100 & 100 \\
\hline & distort2-set1 & $5 \times 5$ & 8 & 100 & 100 & 100 & 100 \\
\hline & distort3-set1 & $5 \times 5$ & 8 & 100 & 100 & 100 & 100 \\
\hline & distort1-set2 & $15 \times 5$ & 8 & 100 & 100 & 100 & 100 \\
\hline & distort2-set2 & $15 \times 5$ & 4 & 100 & 100 & 100 & 100 \\
\hline & distort3-set2 & $15 \times 5$ & 4 & 100 & 100 & 100 & 100 \\
\hline \multicolumn{3}{|c|}{ Average - distort } & 7 & 100 & 100 & 100 & 100 \\
\hline \multirow{3}{*}{\begin{tabular}{l}
0 \\
\multirow{0}{0}{} \\
0 \\
0 \\
0 \\
0
\end{tabular}} & set1 & $5 \times 5 \times 9$ & 12 & 86 & 79 & 87 & 95 \\
\hline & set2 & $20 \times 5 \times 9$ & 07 & 93 & 79 & 76 & 96 \\
\hline & set3 & $50 \times 5 \times 9$ & 07 & 89 & 77 & 70 & 93 \\
\hline \multicolumn{3}{|c|}{ Average - degrade } & 08 & 89 & 78 & 77 & 95 \\
\hline
\end{tabular}

as missing parts within the shapes. Fig. 6 shows a few samples of it. In this dataset, we aim to retrieve all 300 instances for every chosen query.

Table 2 shows the average retrieval rate for all requested short-lists (e.g., top-20, top-40 and so on). Up to top-60, one cannot make decision that which method performs well since there exists no notable differences in retrieval rate. It is only determined after top-60. To be precise, the aim of the test is to evaluate retrieval stability of the methods. $\mathcal{D}$-Radon provides the rate of $86 \%$ in top-300 while more than $16 \%$ difference (fairly in large amount) has been made with SC. SC lags GFD by approximately $9 \%$. $\mathcal{R}$-signature provides an average results compared to Zernike. Compared to all, $\mathcal{D}$-Radon outperforms.

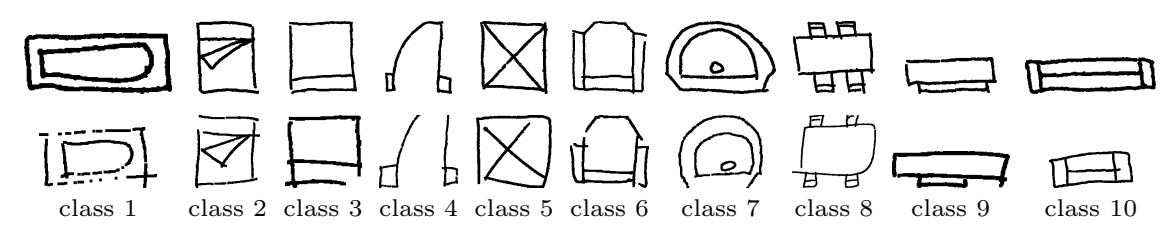

Fig. 6. 2 hand-drawn samples from 10 different known classes.

\section{B. Shape Retrieval}

We have used two different shape datasets [26]: Kimia's Shapes99 and Shapes216. Shapes99 dataset consists of 9 classes, each one is having 11 samples. In another dataset, there are 18 classes and each one contains 12 samples. Fig. 7 shows a few 
Table 2. Average retrieval rate in $\%$ for $C V C$ hand-drawn symbol dataset

\begin{tabular}{|lcccccccccccccccc|}
\hline & Test & top & top & top & top & top & top & top & top & top & top & top & top & top & top & top \\
& images & 20 & 40 & 60 & 80 & 100 & 120 & 140 & 160 & 180 & 200 & 220 & 240 & 260 & 280 & 300 \\
\hline \hline Zernike & & 48 & 42 & 39 & 37 & 35 & 34 & 33 & 32 & 30 & 30 & 29 & 28 & 28 & 27 & 26 \\
$\mathcal{R}-$ sign. & $\&$ & 82 & 75 & 69 & 65 & 62 & 59 & 56 & 54 & 51 & 49 & 48 & 46 & 45 & 43 & 42 \\
GFD & $\infty$ & 96 & 93 & 90 & 88 & 85 & 83 & 81 & 78 & 76 & 73 & 71 & 68 & 66 & 63 & 61 \\
SC & $\circ$ & 98 & 95 & 95 & 92 & 91 & 88 & 87 & 85 & 83 & 81 & 78 & 78 & 75 & 73 & 70 \\
$\mathcal{D}-$ Radon & & $\mathbf{9 9}$ & $\mathbf{9 9}$ & $\mathbf{9 8}$ & $\mathbf{9 7}$ & $\mathbf{9 7}$ & $\mathbf{9 6}$ & $\mathbf{9 5}$ & $\mathbf{9 4}$ & $\mathbf{9 3}$ & $\mathbf{9 2}$ & $\mathbf{9 1}$ & $\mathbf{9 0}$ & $\mathbf{8 9}$ & $\mathbf{8 7}$ & $\mathbf{8 6}$ \\
\hline
\end{tabular}

(a) shapes99
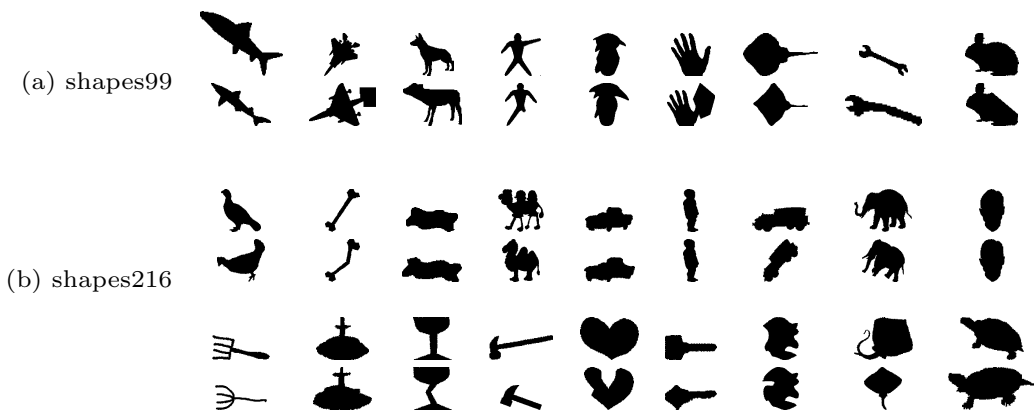

Fig. 7. 2 samples from each class of (a) shapes99 and (b) shapes216 datasets.

samples of both datasets. As in [26], we have used the dataset for recognition purpose. For retrieval rate, since there are $N$ instances from each class, we have increased proximity search from 1 to $N$ with the step of +1 . In addition, we have tested Bull's eye score [21,4]. Bull's eye score is the ratio of the number of correct matches up to proximity search space of $2 N$ to the possible number of matches in the dataset.

Table 3 shows the experimental results for both datasets. Compared to Zernike and $\mathcal{R}$-signature, our method outperforms with significant difference while it goes almost equally with GFD and SC.

\section{Discussions}

We analyse behaviour of the methods based on the key characteristics as well as major challenges of test images. In graphical symbol datasets, samples are distorted, embedded with different levels of noise and even degraded. In particular, there exists missing parts including severe vectorial distortions in hand-drawn symbol dataset in addition to a significant size variation as well as multi-class similarity between the classes. Besides, occlusion exists more in hand-drawn and shape datasets.

Of course, global signal based descriptors are easy to implement as explained in Section 1. However, they are generally not well adapted for capturing precisely small detail changes as well as this tends to make them fault tolerant to image distortions, noise as well as missing parts. We have examined such a behaviour throughout the tests. Considering GREC2003 dataset for instance, for 
Table 3. Average retrieval rate and Bull's eye score in $\%$ for kimia's dataset

\begin{tabular}{|c|c|c|c|c|c|c|c|c|c|c|c|c|c|c|c|}
\hline & $\begin{array}{c}\text { Test } \\
\text { image }\end{array}$ & $\begin{array}{c}\text { top } \\
1\end{array}$ & $\begin{array}{c}\text { top } \\
2\end{array}$ & $\begin{array}{c}\text { top } \\
3\end{array}$ & $\begin{array}{c}\text { top } \\
4\end{array}$ & $\begin{array}{c}\text { top } \\
5\end{array}$ & $\begin{array}{c}\text { top } \\
6\end{array}$ & $\begin{array}{c}\text { top } \\
7\end{array}$ & $\begin{array}{c}\text { top } \\
8\end{array}$ & $\begin{array}{c}\text { top } \\
9\end{array}$ & $\begin{array}{c}\text { top } \\
10\end{array}$ & $\begin{array}{c}\text { top } \\
11\end{array}$ & $\begin{array}{l}\text { top } \\
12\end{array}$ & $\begin{array}{l}\text { bull's eye } \\
\text { score }\end{array}$ \\
\hline \multirow{5}{*}{ 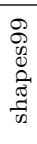 } & Zernike & \multirow{5}{*}{$\begin{array}{l}\vec{\exists} \\
\stackrel{x}{\sigma}\end{array}$} & 100 & 74 & 66 & 59 & 53 & 48 & 45 & 42 & 40 & 37 & 35 & & 48 \\
\hline & $\mathcal{R}-$ sign & & 100 & 83 & 73 & 66 & 60 & 56 & 51 & 49 & 47 & 45 & 43 & & 59 \\
\hline & $\mathrm{SC}$ & & 100 & 97 & 95 & 92 & 89 & 87 & 85 & 84 & 82 & 80 & 77 & & 84 \\
\hline & GFD & & 100 & 97 & 96 & 92 & 90 & 87 & 83 & 80 & 77 & 73 & 70 & & 86 \\
\hline & $\mathcal{D}$-Radon & & 100 & 97 & 95 & 94 & 92 & 91 & 86 & 81 & 80 & 79 & 77 & & 87 \\
\hline \multirow{5}{*}{ 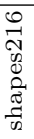 } & Zernike & \multirow{5}{*}{$\begin{array}{l}\stackrel{\sim}{\sim} \\
\underset{\sim}{x} \\
\underset{\sim}{-1}\end{array}$} & 100 & 81 & 71 & 63 & 57 & 53 & 50 & 48 & 45 & $\overline{43}$ & 41 & 39 & 48 \\
\hline & $\mathcal{R}-\operatorname{sign}$ & & 100 & 86 & 80 & 76 & 71 & 67 & 65 & 62 & 59 & 57 & 54 & 52 & 64 \\
\hline & GFD & & 100 & 97 & 93 & 91 & 87 & 85 & 84 & 81 & 79 & 78 & 76 & 73 & 83 \\
\hline & $\mathrm{SC}$ & & 100 & 99 & 97 & 95 & 93 & 91 & 90 & 89 & 88 & 86 & 83 & 80 & 87 \\
\hline & $\mathcal{D}$-Radon & & 100 & 99 & 95 & 93 & 91 & 90 & 89 & 86 & 85 & 84 & 83 & 81 & 88 \\
\hline
\end{tabular}

degradation models $\mathcal{R}$-signature is affected from every dot (from noise) due to the square effect (via $\mathcal{R}$-transform). Similarly, SC is affected where the level of noise (see $\mathrm{m} 2$ to $\mathrm{m} 7$ in Fig. 5) is high since it accounts those pixels while sampling. For Zernike, the disadvantage (in particular if high degree polynomials are involved) is the unequal distribution of nodal lines over the unit disk. We have observed the effect in case of degradation models: $\mathrm{m} 8$ and $\mathrm{m} 9$ in Fig. 5. Similar situations occur in the $C V C$ hand-drawn symbols. GFD on the whole, provides average results. Those descriptors provide interesting results for silhouette shapes. Considering such datasets, our method performs reasonably better. More specifically, it can optimally handle noisy, degraded as well as distorted samples as well as where internal content is necessary to be considered.

Running time complexity is usually high since it uses DTW for matching radon histograms, however it is largely depend on how big the image is. As far as concern to computational cost, the observed average running time for all methods is given in Table 4. We have used MATLAB 7.8.0 in Linux platform.

Table 4. Average running time for generating features and matching for a single pair.

\begin{tabular}{|c|c|c|c|c|c|}
\cline { 2 - 6 } \multicolumn{1}{c|}{} & $\mathcal{R}$-sign. & GFD & Zernike & SC & $\mathcal{D}$-Radon \\
\hline Time (sec.) & 1.5 & 15 & 20 & 43 & 77 \\
\hline
\end{tabular}

\section{Conclusions}

We have presented a method for graphics recognition under document analysis and shape retrieval under CBIR applications. The method is basically based on dynamic programming for matching radon features. The method is quite simple and easy to implement since it is parameter free.

Computing radon transform is quite immediate. But the running time complexity lies in matching. However, it could be substantially reduced by using optimised DTW [25] - a step to go further. 


\section{References}

1. Attalla, E., Siy, P.: Robust shape similarity retrieval based on contour segmentation polygonal multiresolution and elastic matching. PR 38(12), 2229-2241 (2005)

2. Bailey, R.R., Srinath, M.: Orthogonal moment features for use with parametric and non-parametric classifiers. IEEE PAMI 18(4), 389-399 (1996)

3. Bandera, C.U.A., Sandoval, F.: Non-parametric planar shape representation based on adaptive curvature functions. PR 35, 43-53 (2002)

4. Belongie, S., Malik, J., Puzicha, J.: Shape matching and object recognition using shape contexts. IEEE PAMI 24(4), 509-522 (2002)

5. Bernier, T., Landry, J.A.: A new method for representing and matching shapes of natural objects. PR 36(8), 1711-1723 (2003)

6. Boulgouris, N.V., Chi, Z.X.: Gait recognition using radon transform and linear discriminant analysis. IEEE Image Processing 16(3), 731-740 (2007)

7. Bunke, H., Riesen, K.: Recent advances in graph-based pattern recognition with applications in document analysis. PR 44(5), 1057-1067 (2011)

8. Chong, C., Raveendran, P., Mukudan, R.: A comparative analysis of algorithms for fast computation of zernike moments. PR 36, 731-742 (2003)

9. Coetzer, J.: Off-line Signature Verification. Ph.D. thesis, University of Stellenbosch (2005)

10. Conte, D., Foggia, P., Sansone, C., Vento, M.: Thirty years of graph matching in pattern recognition. IJPRAI 18(3), 265-298 (2004)

11. Deans, S.R.: Applications of the Radon Transform. Wiley Interscience Publications, New York (1983)

12. El-ghazal, A., Basir, O., Belkasim, S.: Farthest point distance: A new shape signature for fourier descriptors. Signal Processing: Image Communication 24(7), 572$586(2009)$

13. Flusser, J.: On the independence of rotation moment invariants. PR 33(9), 1405$1410(2000)$

14. Fränti, P., Mednonogov, A., Kyrki, V., Kälviäinen, H.: Content-based matching of line-drawing images using the hough transform. IJDAR 3(2), 117-124 (2000)

15. GREC: International symbol recognition contest at grec2003 (2003), http://www . cvc.uab.es/grec2003/SymRecContest/

16. Jafari-Khouzani, K., Soltanian-Zadeh, H.: Radon transform orientation estimation for rotation invariant texture analysis. IEEE PAMI 27(6), 1004-1008 (2005)

17. Jain, A.K., Duin, R.P.W., Mao, J.: Statistical pattern recognition: A review. IEEE PAMI 22(1), 4-37 (2000)

18. K.C., S., Wendling, L., Lamiroy, B.: Using spatial relations for graphical symbol description. In: ICPR. pp. 2041-2044 (2010)

19. Kim, W.Y., Kim, Y.S.: A region-based shape descriptor using zernike moments. Signal Processing: Image Communication 16(1-2), 95-102 (2000)

20. Kruskall, J.B., Liberman, M.: The symmetric time warping algorithm: From continuous to discrete. In: Time Warps, String Edits and Macromolecules: The Theory and Practice of String Comparison. pp. 125-161. Addison-Wesley (1983)

21. Latecki, L.J., Lakmper, R., Eckhardt, U.: Shape descriptors for non-rigid shapes with a single closed contour. In: CVPR. pp. 1424-1429 (2000)

22. Leavers, V.: Use of the two-dimensional radon transform to generate a taxonomy of shape for the characterization of abrasive powder particles. IEEE PAMI 22(12), 1411-1423 (2000)

23. Loncaric, S.: A survey of shape analysis techniques. PR 31(8), 983-1001 (1998) 
24. R., J., Kolhe, S.R., Patil, P.M.: Dynamic time warping based static hand printed signature verification. PRR 4(1), 52-65 (2009)

25. Salvador, S., Chan, P.: Toward accurate dynamic time warping in linear time and space. Intell. Data Anal. 11(5), 561-580 (2007)

26. Sebastian, T.B., Klein, P.N., Kimia, B.B.: Recognition of shapes by editing shock graphs. In: ICCV. pp. 755-762 (2001)

27. Tabbone, S., Wendling, L., Salmon, J.P.: A new shape descriptor defined on the radon transform. CVIU 102(1), 42-51 (2006)

28. Teh, C.H., Chin, R.T.: On image analysis by the methods of moments. IEEE PAMI 10(4), 496-513 (1988)

29. Wendling, L., Rendek, J., Matsakis, P.: Selection of suitable set of decision rules using choquet integral. In: SSPR/SPR. pp. 947-955 (2008)

30. Zhang, D., Lu, G.: Shape-based image retrieval using generic fourier descriptor. Signal Processing: Image Communication 17, 825-848 (2002)

31. Zhang, D., Lu, G.: Review of shape representation and description techniques. PR 37(1), 1-19 (2004)

32. Zhang, D., Lu, G.: Study and evaluation of different fourier methods for image retrieval. IVC 23(1), 33-49 (2005)

33. Zhu, S.C., Yuille, A.L.: Forms: A flexible object recognition and modelling system. IJCV 20(3), 187-212 (1996) 